\title{
Médiévales
}

Langues, Textes, Histoire

\section{Le livre «L'arbre-mirage » et la réflexion sur les romans dans le Roman du Genji (XI siècle)}

The "Broom-Tree" Book and the Reflexion on the Novel in the Tale of Genji (11th Century)

\section{Daniel Struve}

\section{(2) OpenEdition}

\section{Journals}

Édition électronique

URL : https://journals.openedition.org/medievales/8102

DOI : 10.4000/medievales.8102

ISSN : 1777-5892

Éditeur

Presses universitaires de Vincennes

Édition imprimée

Date de publication : 22 juin 2017

Pagination : 125-144

ISBN : 978-2-84292-612-0

ISSN : 0751-2708

Référence électronique

Daniel Struve, «Le livre «L'arbre-mirage » et la réflexion sur les romans dans le Roman du Genji (x। ${ }^{e}$ siècle) », Médiévales [En ligne], 72 I printemps 2017, mis en ligne le 28 février 2019, consulté le 22 avril 2022. URL : http://journals.openedition.org/medievales/8102 ; DOI : https://doi.org/10.4000/ medievales.8102 
Daniel Struve

\section{Le livre "L'arbre-mirage " et la réflexion sur les romans dans le Roman du Genji (XI ${ }^{\mathrm{e}}$ siècle)}

\section{La situation du roman dans le Japon de l'époque de Heian}

Au tournant des IX $X^{e}$ et $X^{e}$ siècles, le Japon voit l'éclosion d'un roman en japonais dont le chef-d'œuvre est le Roman du Genji, rédigé par une dame de cour, Murasaki Shikibu, au début du $\mathrm{XI}^{\mathrm{e}}$ siècle. Cette œuvre célèbre, devenue un classique de la littérature mondiale, couronne un siècle d'élaboration, de mûrissement et de prolifération du genre romanesque dans les milieux aristocratiques. Cette vogue du roman, dont on sait peu de choses, la plus grande partie des œuvres étant perdues, est attestée par quelques rares témoignages, comme la préface au recueil d'anecdotes bouddhiques intitulé Sanbôe (Illustration des Trois Joyaux) de Minamoto no Tamenori, daté de 984 et destiné à la princesse Sonshi, fille de l'empereur En.yû, qui venait d'entrer en religion ${ }^{1}$. L'auteur y fait éloge du choix qu'a fait la princesse de quitter ce monde évanescent pour s'engager dans la voie de la Loi et la met en garde contre les passe-temps auxquels elle sera tentée de recourir : le jeu de go, la cithare japonaise, et enfin les romans. C'est d'ailleurs pour lutter contre ces distractions et aider la princesse à passer utilement les longues nuits d'automne qu'il lui présente un ouvrage propre à servir à son édification. Voici comment y sont décrits les romans :

Ce qu'on appelle les monogatari, qui distraient le cœur des dames, sont plus nombreux que les herbes sauvages ou que les grains de sable sur les grèves. Ils font parler animaux et plantes qui ne sont pas doués de parole et font éprouver des sentiments à ce qui en est dépourvu. Ils évoquent des

1. Sur Tamenori et Sonshi, voir l'introduction à la traduction anglaise de d'E. KAMENS, The Three Jewels. A Study and Translation of Minamoto Tamenori's « Sanbōe », Ann Arbor, 1988 (Michigan Monograph Series in Japanese Studies vol. 2). 
événements sans plus de fondement qu'un bout de bois ballotté par les vagues, combinent des paroles sans contenu véridique, aussi incertaines que les plants de la zizanie, qui poussent dans l'eau. Iga no tawome, Tosa no otodo, Imameki no Chûjô, Nakai no Jijû, etc., décrivent sans retenue les rapports entre hommes et femmes. Vous ne devez prêter si peu que ce soit attention à ces forêts de paroles, à ces racines de péché2

L'auteur reproche aux romans leur caractère mensonger, le fait qu'ils affirment ce qui n'existe pas dans la réalité. Mais il leur fait également grief de parler d'amour et ainsi de pouvoir séduire un lecteur même aussi bien intentionné que la princesse, d’être des « racines de péchés ».

On trouve un écho de cette prévention, sans doute bien partagée, contre les monogatari chez l'auteur des Mémoires d'une Éphémère, l'épouse du ministre Fujiwara no Kaneie, également de la fin du $\mathrm{X}^{\mathrm{e}}$ siècle. Voici ce qu'on lit dans la préface de l'ouvrage :

Il était une fois une personne - les jours qu'elle avait vécus s'étant enfuis dont la condition en ce monde était fragile, incertaine. Avec un visage qui n'égalait point celui du commun des femmes, dépourvue d'esprit, c'était raison qu'elle menât une vie inutile, se disait-elle, et elle laissait simplement passer les jours, matin après matin, soir après soir ; à jeter les yeux sur les romans anciens qui pullulent de par le monde, il n'en manque pas qui regorgent de sornettes (soragoto) : à plus forte raison, aller faire d'une vie insignifiante le sujet de mémoires paraîtra étrange. Mais j'ai pensé que mon ouvrage pourrait proposer un exemple à ceux qui s'interrogent sur la vie des personnes de la plus haute condition. Au demeurant, comme ce qui touche aux mois, aux années passées, s'est brouillé, il est nombre de choses qui devront rester comme cela ${ }^{3}$.

Ces lignes ont été étudiées en détail par Jacqueline Pigeot dans le commentaire qui accompagne sa traduction de cet ouvrage ${ }^{4}$. On peut notamment remarquer dans le propos de la mère de Michitsuna le terme dépréciatif de « romans anciens » (furumonogatari). Comme pour l'auteur du Sanbôe, pour elle aussi les romans ont tendance à «pulluler » et rapportent des événements sans rapport avec la réalité : des «sornettes » (soragoto), littéralement des «propos vides, sans fondement» (le mot sora signifiant aussi « ciel », comme étendue vide d'objets). Le récit véridique d'une vie

2. Minamoto no Tamenori, Sanbôe, dans Mabuchi K. et al. éd., Sanbôe, Chôkôsen, coll. Shin Nihon Koten Bungaku Taikei, vol. 31, Tôkyô, 1997, p. 6. Traduction anglaise par E. KAMENS, The Three Jewels..., p. 93. Aucun des récits énumérés ici ne nous est parvenu.

3. Mémoires d'une Éphémère (954-974) par la mère de Fujiwara no Michitsuna, trad. J. PIGEOT, Paris, 2006, p. 9.

4. Ibid., p. 197 sq. 
sans intérêt comme la sienne sera peut-être plus insipide encore, concède l'auteur ; mais au moins sera-t-il vrai et donc susceptible d'être de quelque utilité, à condition que la mémoire ne se montre pas trop défaillante. Du reste, le propos de l'auteur des Mémoires d'une Éphémère ne manque pas d'être ambigu. Le discrédit dont semble avoir souffert le genre du monogatari ou récit de fiction à l'époque de Heian, ou du moins une partie de cette production identifiée comme «ancienne »(furumonogatari), n'empêche pas le roman d'être pris comme point de départ et de référence. Ainsi, l'autobiographie prend le contre-pied du roman et s'élabore en opposition à lui, tout en se définissant par rapport à ce genre : les romans occupaient donc une place non négligeable dans le paysage littéraire au tournant des $\mathrm{X}^{\mathrm{e}}$ et $\mathrm{XI}^{\mathrm{e}}$ siècles, du moins dans celui de l'expression en japonais, où à cette époque commencent à dominer les femmes, par opposition à l'expression en chinois, de caractère plus officielle et destinée aux hommes.

Sur ce point, les Notes de chevet de la dame du palais Sei Shônagon ( $\mathrm{XI}^{\mathrm{e}}$ siècle) apportent un témoignage précieux. Ce recueil où l'auteur a noté sans plan préconçu des impressions, des réflexions ou des événements de la vie de la cour, est notamment connu pour ses listes où Sei Shônagon se plaît à réunir autour d'une idée des termes hétéroclites. Parmi ces listes on rencontre les deux suivantes, placées l'une à la suite de l'autre et intitulées respectivement «Choses ennuyeuses » et «Choses pour se distraire de l'ennui » :

\section{$\S 133$ Choses ennuyeuses [tsuredzure naru mono]}

On est retenu loin de chez soi pour observer une période d'abstinence. Au jeu de trictrac, les pions n'avancent pas. Une maison dont le maître n'a pas obtenu de fonction lors de la séance des promotions au Palais. L'ennui est encore plus fort lorsqu'il pleut.

\section{$\S 134$ Choses pour se distraire de l'ennui [tsuredzure nagusamu mono]}

Le jeu de go, le jeu de trictrac, les romans [monogatari]. Un enfant de trois ou quatre ans a un mot charmant. Ou bien encore un très jeune enfant qui babille [...]. Des friandises. Un homme facétieux et sachant parler vient vous rendre visite et, quoiqu'on observe un interdit, vous le laissez entrer ${ }^{5}$.

Ces brèves remarques de Sei Shônagon sont très suggestives pour bien comprendre la place particulière occupée par le roman dans la société de

5. Sei Shonagon, Makura no sôshi, éd. Matsuo S., Nagai K., coll. Shinpen Nihon Bungaku Zenshû, Tôkyô, 1997, p. 253-254. Le passage signalé par des crochets correspond à une expression dont le sens est inconnu. Dans la traduction des Notes de chevet d'André Beaujard, ces deux chapitres sont traduits respectivement comme "Choses qui remplissent l'âme de tristesse » et "Choses qui distraient dans les moments d'ennui » (§ 63 et 64) : SEI SHÔNAGON, Notes de chevet, trad. A. BEAUJARD, Paris, 1966, p. 161. 
l'époque de Heian. Elles montrent le lien qui existe dans l'esprit de l'auteur entre le sentiment d'ennui et certains moments particuliers d'inactivité forcée, comme les périodes d'abstinence rituelles, les situations d'échec ou, plus banalement, les jours de pluie, mais aussi avec certaines formes de distraction dont font partie les romans (monogatari) et, plus largement, l'art de bien parler (mono ihu). Le terme tsuredzure, nom ou adjectif selon le contexte, décrit le sentiment éprouvé dans une situation où l'action est impossible, et où par conséquent la fiction peut se déployer. On peut considérer ainsi que les récits romanesques se sont développés en marge de la littérature sérieuse, poésie et prose en chinois ou poésie en japonais. Certes, le roman se situe tout en bas de la hiérarchie officielle des genres, mais il occupe un domaine propre, celui des activités distrayantes, qui possèdent leur propre hiérarchie de valeurs et leurs propres critères d'excellence. Comme le montre le témoignage de Sei Shônagon, certains membres de la société aristocratique étaient reconnus pour leur capacité à «bien parler» (mono ihu), talent qui leur conférait le statut de « beaux parleurs » (mono-ihi) et qui faisait que leur présence était particulièrement appréciée pendant les moments d'inaction et d'ennui (tzuredzure). D'autres encore, comme Murasaki Shikibu, excellaient dans la composition de romans, qui pouvaient jouer une fonction analogue.

\section{Le « débat sur les romans »}

Dans le livre intitulé «Les lucioles » du Roman du Genji se trouve un passage fameux ${ }^{6}$ qui aborde la question du statut et du rôle des romans. L'action se passe au moment des pluies du cinquième mois (samidare), saison de désœuvrement et de confinement à l'intérieur des maisons, caractérisée par ce sentiment de vide et d'ennui que désigne le terme de tsuredzure. Le Genji rend successivement visite à sa fille adoptive, dame Tamakazura, qu'il cherche à marier avantageusement tout en la poursuivant de ses assiduités, puis à son épouse favorite, dame Murasaki. Toutes les deux sont des passionnées de romans. Tamakazura, la plus enthousiaste des deux, est occupée à en recopier. Elle cherche en même temps à retrouver parmi les héroïnes de ces histoires celles dont le sort se rapprocherait du sien. Le Genji la trouve plongée dans cette occupation, ce qui lui arrache une remarque ironique :

6. Sur ce passage voir J. PIGEOT, « Le système de lecture de Motoori Norinaga (17301801) », dans Repenser l'ordre, repenser l'héritage. Paysage intellectuel du Japon (XVII'XIX $x^{e}$ siècles), éd. F. GIRARD et al., Genève, 2002, p. 315 sq. Cf. aussi F. LAVOCAT, Fait et Fiction. Pour une frontière, « Poétique », Paris, 2016, p. 189 sq. (chapitre «Penser la fiction dans le Dit du Genji »). 
Le Ministre qui, chez les unes et chez les autres trouvait sans cesse pareils écrits éparpillés, lui dit un jour :

- C'en est exaspérant ! À croire que les femmes sont nées pour se laisser duper ${ }^{7}$.

S'ensuit une longue conversation, connue comme le « débat sur les romans » (monogatari ron). Si le Genji rabaisse d'abord les romans en les qualifiant de « sornettes » (suzurogoto) tout juste bonnes à donner le change à de naïves lectrices féminines, il se ravise ensuite et, quoiqu'en plaisantant, fait l'éloge de l'habileté avec laquelle ces récits savent captiver leurs lecteurs, y compris un courtisan lettré, formé aux lettres chinoises, comme lui. Au moins, admet-il, leur faut-il reconnaître le mérite de «distraire des moments d'ennui » :

À dire vrai, s'il n'était ces vieilles histoires, comment pourriez-vous vous distraire de l'inévitable ennui [tsuredzure] de cette saison ? Parmi ces inventions [itsuhari] il en est, je le veux bien, qui montrent si bien les sentiments, que l'on se dit qu'en vérité cela se pourrait, et pour peu que le récit se déroule de façon vraisemblable, l'on a beau savoir que tout cela n'a point le sens commun [hakanashigoto], on se laisse émouvoir sans la moindre raison ; et si nous y voyons se tourmenter quelque charmante demoiselle, une part de notre esprit s'y laisse prendre ! Tel autre, dont l'invraisemblance saute aux yeux, et qui n'est qu'un tissu d'extravagances, nous surprend tout d'abord, puis nous irrite quand nous l'écoutons une seconde fois la tête reposée, et pourtant il peut s'en trouver qui nous procurent un plaisir évident. Ces temps-ci il m'est arrivé d'en entendre que la jeune demoiselle se faisait lire par ses femmes, et je me disais que décidément il existait des gens qui savaient adroitement dire les choses [mono yoku ihu], et que s'ils s'exprimaient si bien, ce devait être grâce à l'aplomb que donne une grande habitude du mensonge [soragoto], mais sans doute n'est-ce pas encore tout à fait cela ${ }^{8}$ !

Une sorte de charme agit sur le lecteur de roman. Entraîné par l'enchaînement des événements, il est amené à s'intéresser à des faits et à des personnages dont il sait qu'ils sont inventés, mais qui lui paraissent néanmoins sortis de la réalité. Le Genji admire l'éloquence, l'habileté à manier le langage (mono yoku ihu) dont font preuve les auteurs de ces récits, la virtuosité avec

7. MURASAKI ShIKIBU, Genji monogatari, t. 3, coll. Shinpen Nihon Bungaku Zenshû, vol. 22, Tôkyô, 1996, p. 210 : trad. R. SiefFert, MurasaKi SHIKIBU, Le Dit du Genji, Paris, 1988 , t. I, p. 506.

8. Ibid., p. $211 ;$ p. 506-507. 
laquelle ils tiennent des «mensonges » ou, si l'on traduit plus littéralement, des « propos creux, sans fondement» (soragoto).

Tamakazura rappelle alors au Genji ses propres mensonges et affirme qu'elle lit les romans avec plus de simplicité :

En fait, ne serait-ce pas plutôt ceux qui ont coutume d'en conter à autrui [itsuhari naretaru hito], qui cherchent ainsi la petite bête ? Quant à moi, je prends tout pour argent comptant [makoto no koto] ! dit-elle? .

En somme, chaque lecteur trouve dans les romans ce qu'il y met. De tous les termes employés par le Genji pour qualifier les romans, Tamakazura retient celui d' « invention » ou «mensonge » (itsuhari), comme porteur de l'accusation la plus grave, celle d'une volonté délibérée d'induire en erreur. À la lecture soupçonneuse du Genji, elle oppose sa propre lecture, naïve, d'adhésion spontanée à la fiction, qu'elle prend pour la vérité (makoto) sans se poser la question de son statut ontologique. Cette opposition entre mensonge (itsuhari) et vérité (makoto), introduite par Tamakazura, induit un retournement dans le discours du Genji. Tout en riant, ce dernier cherche maintenant à montrer que les romans, même s'ils présentent une réalité qui n'existe pas, ne sont pas sans contenir des enseignements pour ceux qui sauraient les lire. Les romans nous parlent d'aspects de la vie qu'ignore l'histoire officielle, laquelle se limite aux événements publics. Ils peuvent donc se voir reconnaître une certaine valeur de vérité, quoiqu'indirecte. Pour finir, le Genji compare les romans aux paraboles des écritures bouddhiques, qui enseignent par des moyens détournés (hôben).

Sans procéder à une analyse détaillée de ce passage à juste titre célèbre, nous nous limiterons à quelques remarques. On peut noter tout d'abord que la question des romans, de leur valeur et de leur mode de fonctionnement, est abordée de deux manières différentes. Pour commencer, le Genji adopte le point du vue du récepteur (lecteur ou auditeur), rejetant dans un premier temps les romans comme mensongers, puis louant leur pouvoir de fascination sur les lecteurs. Cependant, dès la fin du premier développement, le Genji vante l'éloquence des auteurs de romans, et c'est du point de vue de l'auteur qu'il aborde la question dans la seconde partie de son discours, après l'intervention de Tamakazura :

Il ne s'agit évidemment pas des aventures de tel personnage racontées dans leur réalité. À force d'observer $[$ miru $]$ inlassablement la manière d'être des hommes qui vivent en ce monde, en bien comme en mal, à force de les écouter $[k i k u]$ avec passion, l'on découvre des choses que l'on aimerait

9. Ibid., p. 211-212; p. 507. 
transmettre à la postérité, et c'est ainsi que, ne les pouvant garder pour soi [kokoro ni kome-gatakute], l'on en vient à les coucher par écrit [ihi-oki ${ }^{10}$.

La portée de ces lignes apparaît pleinement lorsqu'on les considère à la lumière de la tradition littéraire japonaise et de la réflexion sur la poésie waka. En effet, on a pu relever la similitude des termes employés par le Genji pour parler des romans avec ceux dont un siècle auparavant le poète Ki no Tsurayuki usait dans sa préface à la première anthologie de poésie japonaise compilée sur ordre impérial, le Recueil de poèmes japonais anciens et modernes (Kokin wakashû) : "La poésie c'est de laisser s'exprimer son cœur à travers les choses qu'on voit et qu' on entend ${ }^{11}$. De même que le poème a pour origine les impressions produites sur le cœur par le monde extérieur, de même le roman naît d'une accumulation d'observations faites par le romancier, incapable de retenir plus longtemps le poids des impressions qu'il accumule en son for intérieur. Le raisonnement que tient le Genji est le suivant : les événements rapportés dans le roman ont beau être fictifs, ils n'en sont pas pour autant inventés de toutes pièces. S'appuyant sur les perceptions de l'auteur, ils ne sont donc pas étrangers aux réalités du monde réel, mais en sont le reflet passé par le cœur du romancier et retravaillé en vue de l'effet à produire sur le lecteur. Tout est inventé dans le roman, mais en un sens tout y est vrai. Le rôle du lecteur, dans l'approche proposée ici de ces récits, est déterminant, ce qui explique les revirements du discours du Genji.

On a souvent posé la question du statut de ces propos, que le Genji tient en riant dans une scène où lui-même joue le rôle de ces « beaux parleurs »(mono-ihi) dont, selon Sei Shônagon, on appréciait particulièrement les interventions dans les moments d'inaction ou les jours de pluie. S'agit-il d'une opinion du Genji ou de l'auteur, Murasaki Shikibu ? Ou bien s'agit-il d'une façon de penser répandue dans les milieux cultivés de cette époque ? Une réponse univoque à ces questions est impossible dans la mesure où justement le statut du roman se caractérise par l'ambiguïté. La fiction relève du jeu, auquel se prêtent le lecteur comme l'auteur. Elle ne saurait se justifier que sur le ton de la plaisanterie, sauf à perdre son statut de fiction et à retomber du côté du mensonge. On peut dire qu'elle relève d'un pacte particulier entre l'auteur et le lecteur. Le procédé consistant à prêter au Genji un discours qui correspond à la description par la romancière de

10. Ibid.,p. 212 ; p. 507. Ihi-oku, traduit par René Sieffert comme « coucher par écrit », signifie littéralement « dire, laisser un message en vue de quelque chose ». On notera que l'art du romancier, tout comme celui du poète, est décrit à l'aide de termes désignant une énonciation orale : il s'agit de dire (ihu) et non d'écrire (kaku).

11. Le Monument poétique de Heian : le Kokinshû, trad. G. BonNEAU, vol. 1, préface de KI NO TsURAYUKI, Paris, 1933, p. 26-27. Le rapprochement a été signalé par FUJII S. dans son ouvrage Genji monogatari ron (Étude du Roman du Genji), Tôkyô, 2000, p. 79. 
sa propre démarche n'est donc pas un simple artifice de présentation, mais répond à la nature même de ce genre.

Soulignons, pour finir, l'importance accordée aux personnages. Tamakazura et Murasaki s'identifient ou s'opposent à des personnages de romans antérieurs au Roman du Genji, comme le Roman de Sumiyoshi (Sumiyoshi monogatari) ou le Roman de l'arbre creux (Utsuho monogatari). Le Genji lui-même se compare à un personnage de roman, même s'il prétend ne pas pouvoir identifier un modèle particulier. Aux yeux de ces lectrices passionnées des récits romanesques, les personnages, leur comportement, leur caractère, apparaissent comme des êtres réels. On les critique ou on s'identifie à eux, compatissant à leurs malheurs. Ils sont le lieu de la rencontre entre le lecteur et le romancier. C'est, en effet, à travers les personnages et leur manière d'être au monde que le romancier donne forme au fruit de son observation.

\section{«L'arbre-mirage »}

L'épisode du « débat sur les romans » dans le livre « Les lucioles » témoigne du degré de maturité auquel étaient parvenus l'art romanesque et la réflexion sur le roman dans le milieu aristocratique de l'époque de Heian au début du $\mathrm{XI}^{\mathrm{e}}$ siècle. Le Roman du Genji est l'aboutissement et la somme de cette longue et riche tradition romanesque. Un rôle particulièrement important y est dévolu au deuxième livre, intitulé «L'arbre-mirage »

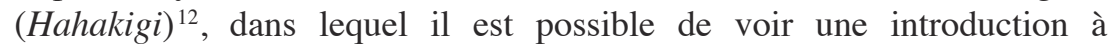
l'ensemble du roman. Il fait suite au premier livre, intitulé « Le clos au paulownia »(Kiritsubo), où sont évoquées la naissance et l'enfance du héros et qui fait plutôt office de prologue. Certes, le livre «L'arbre-mirage » ne présente pas, comme «Les lucioles », une théorie du roman ou un débat sur les romans. Nous y trouvons cependant une représentation figurée de la narration romanesque, qui n'est pas moins importante pour qui veut connaître l'idée que l'auteur du Roman du Genji se faisait de son art. Dans le premier livre, « Le clos au paulownia », l'auteur se contentait d'une brève formule d'introduction - le célèbre « en quel règne était-ce donc ? » - qui signale au lecteur qu'il aborde une chronique fictive, impossible à situer précisément dans le temps historique. Le second livre se caractérise par un dispositif particulièrement élaboré et complexe, une véritable théorie du roman en images. C'est ce dispositif que nous nous proposons de décrire brièvement en le mettant en relation avec le livre « Les lucioles ».

12. Ou «L'arbre-balai » dans la traduction de René Sieffert, puisque tel est le sens littéral de ce mot. L'arbre en question est un arbre imaginaire, évoqué dans la poésie waka, qui disparaît lorsqu'on s'approche de lui. 
Le livre «L'arbre-mirage » s'ouvre par un propos liminaire qui présente le héros du roman comme un personnage complexe de séducteur soucieux de sa réputation et de l'image qu'il laissera à la postérité, et contraint par conséquent de dissimuler et de limiter ses aventures galantes. Évoquant ensuite sa propension à préférer le palais impérial, qui est aussi le palais de son père, à la demeure du Ministre de la Gauche, son beau-père, la narratrice ${ }^{13}$ introduit le personnage de Tô no Chûjô, fils du Ministre et donc beau-frère du Genji, dont il est particulièrement proche. Marié à la fille du Ministre de la Droite, Tô no Chûjô connaît une situation semblable à celle de son ami. Lui aussi évite pareillement la demeure de son épouse. Nous voyons ensuite - et c'est le début du récit proprement dit - les deux amis au palais s'entretenir au cours d'une journée pendant la saison des pluies. La conversation tourne autour de la correspondance galante du Genji, dont Tô no Chûjô tente en vain de se faire révéler le contenu : Tô no Chûjô se réfugie alors dans un propos général sur les femmes : aucune à son avis n'est parfaite. Deux jeunes gens, fonctionnaires de rang moyen, se joignent à la conversation : c'est l'épisode connu comme la « discussion par une nuit de pluie sur les différentes catégories de femmes » (amayo no shinasadame). Un des jeunes gens, le Chef des écuries de la Gauche, beau parleur particulièrement en verve, expose longuement ses théories, soulignant l'intérêt des femmes de catégorie moyenne. Puis chacun y va de son récit : quatre histoires sont alors racontées, deux par le Chef des écuries de la Gauche, une par l'ami du Genji, le Général en second chef de la Chancellerie privée (Tô no Chûjô), et une par le second jeune homme, Adjoint au département des rites, ce qui donne à la première partie du chapitre l'aspect d'un recueil de nouvelles. La conversation se poursuit toute la nuit.

Le lendemain, la pluie cesse, le soleil revient, et le Genji quitte le palais pour la demeure de sa femme, où il ne s'attarde guère. Apprenant l'existence d'un interdit de direction ${ }^{14}$, il se rend chez un de ses obligés, le

13. Le narrateur du Roman du Genji n'apparaît jamais comme un personnage du récit et ne se manifeste que par ses jugements de valeur ou des marques d'énonciation, notamment modales ou relevant des expressions honorifiques (la langue japonaise ignore la catégorie grammaticale de personne). L'ensemble de ces marques, mais surtout l'association étroite de Murasaki Shikibu avec son œuvre, font que le lecteur du Roman du Genji imagine une narratrice appartenant comme Murasaki Shikibu à l'aristocratie de rang intermédiaire, celle dont étaient souvent issues les dames du palais ou les dames de compagnies dans les maisons des grands dignitaires. Il est aussi à noter que le japonais ne fait pas de distinction entre « narrateur » et « narratrice ».

14. Dans le système des croyances du Japon ancien, certaines directions jugées néfastes pouvaient être frappées d'interdit et devaient être contournées. Voir B. FRANK, Kata-imi et kata-tagae. Étude sur les interdits de direction à l'époque Heian, Paris, 1998 («Bibliothèque de l'Institut des hautes études japonaises »). Ces pratiques d'évitement sont souvent utilisées dans les romans, où elles fournissent l'occasion de nombreuses rencontres amoureuses. 
gouverneur de Kii, et passe la nuit chez lui. Justement, la jeune belle-mère de son hôte loge près de ses appartements. Il la rejoint et la force à passer la nuit avec lui. La jeune femme, consciente de l'impossibilité de cette liaison avec un homme bien au-dessus de sa condition, ne lui témoigne que de la froideur. Par la suite, le Genji cherche en vain un moyen de la rejoindre à nouveau, comptant pour cela sur les services de son jeune frère. Il réussit bien à passer une seconde fois la nuit dans la résidence du gouverneur de $\mathrm{Kii}$, mais cette fois la dame est sur ses gardes et se montre inflexible.

Le livre peut être divisé en deux grandes parties : la discussion par une nuit de pluie, puis l'aventure galante du Genji chez le gouverneur de Kii, elle-même constituée de deux moments : la rencontre nocturne, puis les vains efforts que le Genji fait pour renouveler l'occasion au cours des jours qui suivent. On voit que les deux parties sont liées par la continuité chronologique : la rencontre avec Utsusemi a lieu au lendemain de la discussion pendant la nuit de pluie au palais, sans qu'il y ait de coupure temporelle entre ces ceux principaux épisodes. Il s'écoule ensuite un nombre indéterminé de jours, jusqu'à une nouvelle nuit que le Genji passe chez le gouverneur de Kii, mais cette fois en vain. Le livre forme ainsi un ensemble clos et autosuffisant. L'épisode avec la belle-mère du gouverneur de Kii peut constituer un pendant parfait aux quatre histoires racontées au cours de la discussion pendant la nuit de pluie : il met en scène le même type d'héroïne de catégorie intermédiaire dont il est question au cours de la discussion, et comme les autres histoires tourne autour du même motif du malentendu entre amants. Les cinq histoires du livre «L'arbre-mirage » se terminent par des échecs, ce qui confère à leurs héros un caractère quelque peu comique. Utsusemi a beau être séduite par la beauté et les manières du Genji, elle comprend qu'elle n'a rien à gagner, mais au contraire tout à perdre à une telle liaison et regrette de ne pas avoir rencontré le Genji avant son mariage. Elle entre ainsi parfaitement dans la série des héroïnes des contes racontés pendant la nuit de pluie, qui toutes connaissent des déboires dans leurs relations amoureuses, dont certains se révèlent tragiques. Cependant, le livre «L'arbre-mirage » n'est pas non plus un ensemble fermé. Des liens étroits le rattachent aux livres suivants. Un premier ensemble est formé par les trois livres «L'arbre-mirage », « La mue de la cigale » et « La belle-du-soir », puisque l'histoire d'Utsusemi, commencée au second livre («L'arbre-mirage »), se poursuit au troisième («La mue de la cigale ») avec une nouvelle visite, qui porte justement le nom de cette héroïne, tandis que le quatrième livre, « La belle-du-soir », prolonge l'histoire racontée par Tô no Chûjô pendant la nuit de pluie. Ce quatrième livre est clôturé par un bref propos du narrateur qui fait pendant à l'incipit du livre «L'arbre-mirage ». D'autres liens relient ces différents livres au reste de l'œuvre, notamment aux livres qui constituent le cycle de Tamakazura, fille de Belle-du-soir et de Tô no Chûjô. Ainsi, aussi bien les trois premiers livres pris solidairement 
que le seul livre «L'arbre-mirage » pris à part peuvent être considérés comme une introduction au reste de l'œuvre, une entrée en matière et une démonstration de l'art romanesque.

Les correspondances du livre «L'arbre-mirage » avec le livre «Les lucioles » ont notamment été relevées par Fujii Sadakazu ${ }^{15}$. Dans les deux livres, le cadre temporel est le même, celui de ces moments d'ennuis (tsuredzure) amenés par la saison de pluies qu'on peut meubler soit en recopiant, en lisant ou en se faisant lire des romans, soit par des conversations. L'éloquent Chef des écuries de gauche joue auprès du Genji et de Tô ni Chûjô un rôle de beau parleur (mono-ihi) identique à celui que joue le Genji lui-même lors de sa conversation avec Tamakazura dans le livre «Les lucioles ». Dans l'un des deux livres, il s'agit de romans, dans l'autre, de femmes; mais nous l'avons vu, les deux thèmes sont étroitement liés. Il est ainsi question d'héroïnes féminines dans le livre «Les lucioles » et, inversement, il est à plusieurs reprises question de romans dans le livre «L'arbre-mirage » : le mot monogatari (« récit», « propos ») y apparaît à quatre reprises ${ }^{16}$. Ce sont d'ailleurs bien des échantillons de situations romanesques que nous proposent les quatre histoires racontées au cours de la nuit de pluie, qu'on peut désigner par le même mot monogatari. À ces quatre histoires on peut en ajouter une cinquième, qui est racontée dans la deuxième partie du livre ainsi que dans le livre suivant et qui a pour héros le Genji, quand bien même - à la différence des autres - celui-ci n'est pas le narrateur de sa propre histoire. D’une manière générale, le genre romanesque apparaît comme point de référence dans le livre « L'arbremirage ». Ainsi, dans le discours du narrateur au début du livre, le Genji est comparé à un personnage de roman, le capitaine Katano, modèle des séducteurs, à côté duquel il fait pâle figure. Ce propos semble trouver un écho dans le propos tenu par le Genji lorsqu'il imagine quel «personnage d'imbécile » (shiremono) il ferait si son histoire devait faire l'objet d'un roman. Le même terme d' « imbécile » (shiremono) est employé par Tô no Chûjô qui qualifie le récit qu'il s'apprête à faire comme l' « histoire d'un imbécile » (shiremono no monogatari).

Tous ces éléments conduisent à lire les deux livres « L'arbremirage » et «Les lucioles » en regard. Au demeurant, si le roman n'est pas en tant que tel le sujet du débat sur les femmes, la scène de conversation nocturne au cours de laquelle le Chef des écuries de gauche développe ses théories, illustrées ensuite par quatre récits et contenant elles-mêmes des éléments romanesques, peut être lue comme une représentation de la communication romanesque. À plusieurs reprises, pendant les pauses du

15. FUJII S., Genji monogatari ron..., p. 52-83.

16. Dans les expressions : nyôbô no monogatari (récits de femmes), mi-monogatari (récit de vie), shiremono no monogatari (récit d'un fou), mukashi monogatari (récit d'autrefois). 
discours ou entre les histoires, notre attention est attirée en direction des réactions de l'auditoire. Il y a en tout sept interruptions où sont décrites les différentes réactions des auditeurs : commentaires entendus ou gênés de l'Adjoint au département des rites, attention fascinée de Tô no Chûjô, ironie et détachement feint du Genji qui, nous dit-on, ne cesse de penser pendant tout ce temps à celle qu'il tient pour la femme idéale, l'épouse impériale Fujitsubo, dont il est secrètement amoureux.

Voici la première de ces interruptions :

Disant cela, il regardait en direction de l'Adjoint du département des rites qui, se demandant si ces propos ne visaient pas ses propres sœurs cadettes à la réputation flatteuse, resta coi. Le Genji, lui, semblait songeur : même, dans la classe que l'on considérait comme la plus élevée, une femme idéale était chose bien rare, en ce bas monde ${ }^{17}$.

Ou encore :

Ainsi leur prodiguait-il ses mises en garde. Selon son habitude, le Commandant [Tô no Chûjô] acquiesçait. Le Prince esquissa un sourire, comme s'il partageait lui aussi cette opinion : «Qu'il s'agisse de l'une ou de l'autre de vos expériences, elles n'ont rien de glorieux et font peine à entendre », dit-il avant de s'esclaffer, entraînant avec lui toute l'assistance ${ }^{18}$.

Chacun a en tête sa propre expérience qu'il met en rapport avec le contenu du discours ou avec les circonstances des histoires rapportées. Les propos des conteurs trouvent un écho chaque fois différent et entrent en résonnance avec des éléments toujours nouveaux, comme - on peut l'imaginer - le fait aussi le texte d'un roman auprès de ses lecteurs. Le roman ne représente pas un univers fictif séparé de celui de la vie réelle, mais s'insère dans la circulation des discours échangés par les membres de cette société.

Un personnage tient une place particulière dans cette scène. À la première des interruptions citée plus haut, le Genji, seul parmi les assistants, nous est décrit avec quelque détail :

Le Genji, lui, semblait songeur : même dans la classe que l'on considérait comme la plus élevée, une femme idéale était chose bien rare, en ce bas monde... Sur ses vêtements de dessous blancs qui retombaient souplement, il portait juste la casaque de son costume informel, revêtue négligemment,

17. Murasaki ShIKIBU, Genji monogatari, t. 1, p. 61. Pour la traduction française nous citons la traduction du livre «L'arbre-mirage » due au groupe de traduction et d'étude du Roman du Genji du Centre d'études japonaises de l'Inalco, à paraître dans la revue Cipango.

18. Ibid., p. 80-81. 
les cordons dénoués ; confortablement installé, à demi allongé, il était, à la lueur de la lampe, d'une telle beauté que l'on aurait souhaité le voir sous les traits d'une femme. Et pour lui, choisirait-on la femme la plus sublime de la classe la plus élevée, ce choix même jamais ne saurait être satisfaisant ${ }^{19}$.

Nous sommes ici en présence de ce qu'on appelle une intervention du narrateur (sôshiji). Celui-ci (ou celle-ci) intervient pour commenter l'histoire. Le point de vue n'est pas indiqué : il pourrait être celui de n'importe lequel des assistants, mais sans limitation, ce qui permet au lecteur de se transporter en imagination dans le cercle réuni autour d'un si remarquable personnage. Comme les autres personnages, le Genji est décrit uniquement de l'extérieur, mais par un observateur particulièrement attentif et perspicace, capable de déchiffrer les attitudes et les expressions.

Le Genji constitue le second et véritable centre de ce cénacle, occupant une position symétrique par rapport au personnage du Chef des écuries de la Gauche. Autant ce dernier est bavard, autant le Genji est silencieux et en retrait. Il est le seul à ne pas raconter d'histoire, jouant d'un bout à l'autre le rôle d'auditeur. En même temps, c'est lui qui est l'occasion de la réunion et le principal destinataire de tous les discours. L'expérience qu'il fait dès le lendemain d'une tentative de liaison inaboutie avec une de ces femmes de catégorie intermédiaire dont il a été question dans l'entretien, fait explicitement écho aux propos qu'il a entendus et retenus au cours de la soirée. Mais le Genji est aussi le point de référence, le critère incontestable de cet idéal d'élégance et de raffinement dont il est toujours question. Ainsi, on peut voir en lui tout à la fois une figure du lecteur, celui qui écoute les récits et qui, fort des connaissances qu'il a ainsi acquises, va dans un second temps affronter par lui-même la réalité, et une figure de l'auteur, ce point de référence indépassable à partir duquel s'ordonnent les valeurs et les discours.

Personnage paradoxal et problématique, le Genji se caractérise par le secret qu'il porte, et qui, tel une clef de voute, est placé au centre du roman. Le récit y fait à plusieurs reprises allusion, soit indirectement, comme dans la réflexion selon laquelle aucune femme aussi parfaite fût-elle ne semblait pouvoir combler ses désirs, ce qui est une allusion à l'amour secret que le Genji nourrit à l'endroit de l'épouse impériale Fujitsubo, soit plus explicitement, mais toujours de manière voilée, comme à la fin de la série des histoires : 
Ainsi discourait-il, tandis qu'une seule personne ne cessait d'occuper l'esprit du Prince. Chez elle, ni manque, ni excès, elle était vraiment incomparable ! À cette pensée, l'émotion le submergea ${ }^{20}$.

Ou comme c'est le cas lors de la nuit suivante, quand ayant surpris les conversations des femmes dans la chambre voisine de la sienne, le Genji comprend qu'elles parlent de lui et craint un moment qu'elles ne viennent à parler de son secret :

En les entendant chuchoter ainsi, le Prince, pour qui seul comptait son amour, sentit son cœur s'arrêter à l'idée qu'un jour, en de pareilles circonstances, il pourrait surprendre des commérages sur ce qu'il s'évertuait à cacher ${ }^{21}$.

Les thèmes du secret et de la rumeur apparaissent dès les premières lignes du Roman du Genji, qui évoquent les efforts vains du Genji pour garder secrètes ses aventures galantes. Il est repris dans le propos final du livre « La belle-du-soir », où la narratrice présente des excuses sur son indiscrétion, la justifiant par le peu de vraisemblance qu'il y aurait eu à présenter un héros exempt de toute faiblesse, comme il en existe dans les romans, mais pas dans la réalité :

Je m'étais abstenue de divulguer pareilles incartades, puisqu'il cherchait lui-même à les dissimuler à tout prix, mais d'aucuns m'accusaient d'avoir fabriqué cette histoire : pourquoi donc, disaient-ils, sous prétexte qu'il était fils d'Empereur, ceux-là mêmes qui l'ont connu ne feraient-ils que chanter ses louanges comme s'il eût été sans défaut? De toute manière, je n'éviterai pas que l'on me taxe de commérage ${ }^{22}$.

Et c'est toujours ce thème du secret qui est au point de départ de la discussion sur les femmes. La première scène du livre évoque les lettres que le Genji tient serrées dans son coffret et qui suscitent la curiosité de son ami Tô no Chûjo. Document privé et même intime, véhicule privilégié où se disent, souvent sous la forme d'un waka, les sentiments, la lettre est ainsi mise en exergue au tout début des aventures du héros ${ }^{23}$. Celui-ci s'amuse à mystifier son interlocuteur en le mettant au défi d'identifier les auteurs des lettres qu'il lui montre, tout en lui signifiant bien qu'il ne lui montrera jamais

20. Ibid., p. 90-91.

21. Ibid., p. 95.

22. Ibid., p. 195-196 ; trad. R. SIEFFERT, Le Dit du Genji, t. I, p. 92.

23. Le lien de la littérature romanesque en japonais avec le style épistolaire a été souligné par JINNO HIDENORI dans « Nikki bungaku to monogatari-mizukara no kotoba o shobun suru kana bunsho shiron », Kokubungaku, 51/8 (2006), p. 48-56, et dans « Genji monogatari no kotoba to tegami », Bungaku, 7/5 (2006), p. 58-70. 
celles qui pourraient vraiment l'intéresser, c'est-à-dire précisément celles dont le contenu fait la matière du roman. Avec la discussion sur les femmes amorcée aussitôt après par Tô no Chûjô, puis l'audition des différentes histoires, racontées par chacun des participants à l'exception du Genji, nous voyons comment la matière romanesque prend son essor à partir d'un secret, ou plutôt d'un emboîtement de secrets, avec au centre celui, indicible, de l'amour interdit et impossible du Genji pour l'inaccessible Fujitsubo, dont l'image est sans doute répétée dans celles de la « La mue de la cigale » (utsusemi), de la «La belle du soir » (Yûgao), et de «L'arbre-mirage » (hahakigi) censé croître dans les montagnes du Shinano et qui s'évanouit au moment où l'on s'en approche.

\section{L'auteur-narrateur}

Cette évocation de la poétique romanesque de Murasaki Shikibu à travers l'exposition indirecte qu'elle en donne dans le livre « L'arbremirage » serait incomplète sans l'évocation des figures de la narratrice, telles qu'elles apparaissent dans ce livre. La narratrice marque fortement sa présence au tout début du livre :

Prince de lumière : le nom est glorieux, mais la conduite du Genji en ternissait trop souvent l'éclat. Quelle malignité tout de même chez celles qui se plaisaient à rapporter ses aventures, aventures qu'il prenait pourtant soin de garder secrètes [shinobi-tamahi-keru] pour éviter de laisser une réputation de légèreté si le récit en était transmis aux générations à venir ! Au demeurant, il ne lui arrivait plus grand-chose qui fût galant ou charmant, tant il craignait au plus haut point le jugement d'autrui et s'efforçait de paraître irréprochable [mamedachi-tamahi-keru] : le Capitaine de Katanoaurait bien ri de lui !

À l'époque où il était encore Commandant de la Garde, ne se sentant bien que lorsqu'il était en service au Palais, il ne se rendait que de loin en loin à la résidence du Ministre de Gauche. On y avait alors soupçonné quelque «agitation des sentiments », et de fait, si la banalité des liaisons occasionnelles et sans lendemain répugnait à la nature du Prince, il n'en avait pas moins la fâcheuse habitude de s'engager parfois à corps perdu dans des amours malheureuses, ce qui pouvait l'entraîner à des comportements regrettables [uchimajiri-keru] ${ }^{24}$.

Cette présence de la narratrice est marquée de deux manières différentes. D'abord,dans les deux premières phrases, par des terminaisons exclamatives, ainsi que par le suffixe de temps à valeur modale keri, marquant l'ouï-dire,

24. MURASAKI ShIKIBU, Genji monogatari, t. 1, p. 53-54. 
l'explication ou la prise de conscience ${ }^{25}$. La narratrice se présente dans la position de celle qui relate une rumeur, tout en regrettant que celle-ci soit révélée. Ce dispositif fait pendant aux dernières lignes du livre « La belledu-soir » citées plus haut, où la narratrice s'excuse de son indiscrétion. Puis, dans les deux phrases suivantes, par l'emploi du suffixe du passé ki, qui la place dans la même temporalité que le personnage.

Avec le début de l'épisode de la rencontre pendant une nuit de pluie, le roman passe à la narration non marquée, comme si la narratrice s'effaçait devant les événements racontés. Néanmoins elle réapparaît à plusieurs reprises au cours du livre, par exemple dans une remarque telle que celleci :

De nombreux propos fort inconvenants [kiki-nikuki] furent tenus au cours de la dispute ${ }^{26}$.

La narratrice apparaît également à la faveur de modalisations dont certaines ont déjà été relevées lorsque nous avons parlé du point de vue externe adopté dans la scène du débat sur les femmes. En voici encore un exemple :

Des lettres précieuses, qu'il fallait à tout prix cacher, le Prince ne les aurait pas laissé ainsi traîner [uchi-oki-chirashi-bekumoarazu] en désordre dans un coffret si facilement accessible, mais il les aurait rangées en un lieu secret [tori-oki-tamahu-bekamereba]; celles-ci étaient donc certainement [narubeshi] de second ordre, de celles que l'on peut montrer sans que cela prête à conséquence et comme ils en lisaient ensemble quelques passages ${ }^{27} \ldots$

On peut légitimement se demander si les conjectures ne sont pas ici le fait de Tô no Chûjô, qui observe avec curiosité son ami le Genji en train de relire ses lettres ; mais, à l'examen, on peut également les attribuer à la narratrice et les interpréter comme une allusion indirecte au secret du Genji, dont Tô no Chûjô ignore l'existence.

25. Le japonais classique dispose de deux marques de passé non obligatoires, possédant chacune une valeur modale en plus de leur valeur temporelle, et fonctionnant donc comme marques du locuteur. Le suffixe $k i$ marque une action passée située dans la même temporalité que celle du locuteur. Il est notamment employé lorsque le locuteur rapporte des événements dont il a été témoin ou qu'il sait de manière certaine. Le suffixe keri est souvent employé pour les événements du passé appris par ouï-dire, mais peut aussi exprimer une prise de conscience (il perd alors sa valeur temporelle) ou avoir une valeur explicative. Le passé peut également ne pas être marqué. Ces formes non marquées, où n'est pas inscrite la présence du narrateur, sont très fréquentes et peuvent être rapprochées du passé simple français, comme temps du récit au sens de Benvéniste.

26. MurASAKI SHIKIBU, Genji monogatari, t. 1, p. 58.

27. Ibid., p. 55-56. Le suffixe flexionnel beshi, qui apparaît ici trois fois à des formes différentes (en caractère gras), est une marque modale, impliquant le jugement du locuteur. 
Le débat entre le Genji et Tô no Chûjô sur les femmes commence comme un récit à la troisième personne (ou plutôt ànarrateur hétérodiégétique, dans la mesure où la grammaire japonaise ignore la catégorie de personne), adoptant le point de vue du Genji dont les pensées nous sont données à deux reprises :

Le Genji était impressionné en entendant son ami se lamenter ainsi, mais à l'idée que, si lui-même n'avait pas autant de souvenirs de ce genre, il n'en manquait pas non plus, il se prit à sourire ${ }^{28}$.

La curiosité piquée par la maîtrise de la question dont son ami faisait preuve, le Genji lui demanda $[\ldots]^{29}$.

Mais la suite du débat, avec l'arrivée du Chef des écuries de la Gauche et de l'Adjoint du département des rites, marque un retrait de la conscience du Genji. La narratrice se sépare du héros et adopte un point de vue externe, s'interdisant de nous informer sur les pensées du personnage, comme si elle-même se trouvait au milieu des quatre jeunes gens. Ce dispositif ne souffre qu'une seule exception, quand le regard de Tô no Chûjô se pose sur l'Adjoint du département des Rites puis sur le Genji ${ }^{30}$. On peut remarquer que cette partie du livre est aussi celle où la « narration à la $3^{\mathrm{e}}$ personne » (récit à narrateur hétérodiégétique) laisse place aux histoires racontées par les personnages, c'est-à-dire à ce que nous appellerions « narration à la $1^{\text {re }}$ personne » (récit à narrateur intradiégétique). En japonais, cela ne se traduit pas tant par la présence de marques personnelles, même si l'on peut relever des termes désignant le locuteur, mais plutôt par l'emploi d'une marque temporelle spécifique, l'auxiliaire du passé $k i$, dont nous avons vu qu'elle était utilisée au début par la narratrice, et qui retrouve ici tous ses droits puisque chacun des conteurs raconte des événements dont il a été, ou du moins le prétend-il, le témoin ${ }^{31}$. Ces histoires annoncent et préparent

28. Ibid., p. 57.

29. Ibid., p. 58.

30. Il s'agit du passage suivant : « Il regarda alors en direction de l'Adjoint du Département des rites qui resta coi, se demandant sans doute si ces propos ne visaient pas ses propres sœurs cadettes à la réputation flatteuse. "Une femme idéale ? Même parmi celles de la plus haute catégorie, c'est une chose bien rare en ce monde...", devait se dire le Genji. Sur ses vêtements de dessous blancs en soie souple, il portait juste la casaque de son costume informel, revêtue négligemment, les cordons dénoués ; confortablement installé, à demi allongé, il était, à la lueur de la lampe, d'une telle beauté que l'on aurait souhaité le voir sous les traits d'une femme. Et choisirait-on pour lui la dame la plus sublime de la catégorie la plus élevée, ce choix même jamais ne saurait être satisfaisant » (MURASAKI SHIKIBU, Genji monogatari, t. 1, p. 68).

31. Le statut des quatre histoires racontées par le Chef des écuries, l'Adjoint au département des rites et Tô no Chûjô, est ambigu. Toutes hormis celle de Tô no Chûjô 
celle dont le Genji sera le lendemain le héros et qui est prise en charge par la narratrice. Elles ancrent le récit de la narratrice dans l'univers du discours. Il est en particulier à noter que l'histoire de Yûgao (« Belle-dusoir »), d'abord racontée par Tô no Chujô dans le livre «L'arbre-mirage », est poursuivie dans le livre «Belle-du-soir» comme un récit de la narratrice.

On a beaucoup écrit au Japon sur l'identité de la narratrice du Genji, qu'on identifie généralement comme une de ces dames employées au service de grands dignitaires, parfois un peu trop bavardes et enclines à rendre publics les secrets plus ou moins honteux de ses maîtres. Néanmoins, comme cela a été aussi remarqué, la narratrice n'est pas un personnage du roman. Pour autant, elle n'est pas entièrement absente de ce monde, comme l'indiquent les formes de passé certain qu'elle emploie au début du livre (et à d'autres occasions dans le roman). La narration des premiers livres du Roman du Genji semble osciller entre ce que nous appelons une narration à la troisième personne et une narration à la première personne.

Dans ces remarques très incomplètes, nous avons essayé d'aborder la poétique du Roman du Genji à partir des indications mêmes données par l'auteur, que ce soit dans le livre «Les lucioles » ou dans celui de «L'arbremirage». Certes, le livre «L'arbre-mirage » n'est pas à proprement parler un essai sur l'art du roman, mais le dispositif romanesque qu'y met en place l'auteur est particulièrement riche d'enseignement et rejoint le propos sur le roman tenu par le Genji au livre «Les lucioles ». Dans ces deux livres, l'action est située pendant la saison des pluies du cinquième mois, période de vacance propice au récit, qu'il soit oral ou écrit. Le roman se déploie dans ce moment de liberté en marge des occupations de la vie sociale ordinaire et de la vie publique. Autour des personnages romanesques et, singulièrement, du héros porteur de secret qu'est le Genji, se met en place le dialogue indirect entre l'auteur et le lecteur. Le livre « L'arbre-mirage » propose une représentation romanesque de ce dispositif et mérite à ce titre

présentent à des degrés divers un caractère comique et pourraient bien être sinon inventées de toutes pièces, du moins enjolivées, étant manifestement destinées à distraire les personnes présentes. Tout autre est le récit de Tô no Chûjô : la vérité (dans le monde du roman) de l'aventure qu'il raconte est avérée du fait de la réapparition des personnages Belle-du-soir et sa fille Tamakazura dans la suite du roman. On remarque aussi que le récit de Tô no Chûjô conserve d'un bout à l'autre les marques du passé certain $(\mathrm{ki})$, tandis que les autres histoires les abandonnent au moment où le récit parvient au point culminant, le narrateur s'effaçant devant les événements rapportés. Cette différence stylistique est le signe du caractère plus intensément personnel et véridique du récit de Tô no Chûjô. Voir en ligne le texte abrégé de notre communication du 2 juin 2016, « Genji monogatari Hahakigi no maki ni okeru ichininshô katari to sanninshô katari o megutte », sur la page «Atelier : théorie littéraire aujourd'hui » (http://workshop2012paris7.web.fc2.com/yan_jiu_fa_biaodanierusutoryuvu. html). 
d'être lu de près. L'influence de ce livre, et notamment du « débat tenu par une nuit de pluie sur les catégories de femmes » (amayo no shina sadame), a été immense. Et il est la source et sans doute la clef de nombreux procédés de la littérature japonaise jusqu'à l'époque moderne.

Daniel Struve - Université Paris-Diderot; Centre de recherche sur les civilisations d'Asie orientale (CRCAO)

\section{Le livre " L'arbre-mirage " et la réflexion sur les romans dans le Roman du Genji (XI siècle)}

Au Japon, le $X^{e}$ siècle est marqué par l'émergence d'une littérature de fiction en langue vernaculaire, qui se développe dans les milieux de la cour et de l'aristocratie en marge de la culture de langue chinoise et de la poésie en japonais, jouissant toutes deux d'une reconnaissance officielle. Genre marginal et méprisé, le roman ou monogatari, critiqué pour son caractère frivole et son lien avec le mensonge, s'affirme néanmoins comme une activité de distraction propre notamment à meubler les moments d'ennui et d'oisiveté (tsuredzure), comme ceux qu'imposent les pluies du $5^{\mathrm{e}}$ mois. Le Roman du Genji, chef-d'œuvre du genre, rédigé au début du XI ${ }^{\mathrm{e}}$ siècle par la dame de cour Murasaki Shikibu, présente une remarquable réflexion sur la fiction romanesque, que ce soit dans le « débat sur les romans " du livre "Les lucioles " ou dans la "discussion par une nuit de pluie sur les catégories de femmes " qui ouvre le second livre "L'arbre-mirage " et que nous lisons ici comme une mise en scène métaphorique de l'activité romanesque.

Aristocratie de cour - ennui - Japon - langue vernaculaire - roman - Roman du Genji

\section{The "Broom-Tree" Book and the Reflexion on the Novel in the Tale of Genji (11th Century)}

Japan's tenth century is marked by the emergence of literary fiction in the vernacular Japanese language, which gains popularity within the Heian court and the aristocracy alongside the officially recognised Chinese culture and poetry in Japanese (waka), which also had an official status at the court. Though marginal, considered with contempt, and criticised from the perspective of high culture for its frivolity and falsity, the vernacular novel or monogatari establishes itself among the cultural practices of Heian aristocracy as a leisurely activity, strongly connected with moments of vacancy and idleness (tsuredzure) such as the rain season. The masterpiece of this genre, the Tale of Genji written by the court lady Murasaki Shikibu, offers a remarkable reflection on narrative fiction, whether in the "debate on novels" in the chapter entitled "The Fireflies" or in the "discussion about the categories of women on a rainy night" which opens the second chapter "The Broom-Tree" and which we choose to read as a metaphorical staging of novel writing.

Court Aristocracy - Idleness - Japan - Novel - Romance - Tale of Genji Vernacular 
